\section{Muscular dystrophies: key elements for everyday diagnosis and management}

\author{
Alberto Palladino, ${ }^{1}$ Gerardo Nigro, ${ }^{2}$ \\ Luisa Politano' \\ ${ }^{1}$ Cardiomiologia e Genetica Medica - \\ Dipartimento di Medicina Sperimentale; \\ 2Dipartimento di Cardiologia, Seconda \\ Università di Napoli, Italy
}

\section{Abstract}

Muscular dystrophies are a heterogeneous group of inherited disorders that share similar clinical features and dystrophic changes on muscle biopsy, associated with progressive weakness. Weakness may be noted at birth or develop in late adult life. In recent years, cardiac involvement has been observed in a growing number of genetic muscle diseases, and considerable progress has been made in understanding the relationships between disease skeletal muscle and cardiac muscle disease. This review will focus on the skeletal muscle diseases most commonly associated with cardiac complications that can be diagnosed by echocardiography, such as dystrophinopathies including Duchenne (DMD) and Becker (BMD) muscular dystrophies, cardiomyopathy of DMD/BMD carriers and X-L dilated cardiomyopathy.

\section{Introduction}

Muscular dystrophies (MD) are a heterogeneous group of inherited disorders that share similar clinical features and dystrophic changes on muscle biopsy, associated with progressive weakness. ${ }^{1-4}$ Weakness may be noted at birth or develop in late adult life. Some patients manifest with myalgias, rhabdomyolysis, or only raised serum creatine kinase levels without any symptoms or signs of weakness. Early- or childhood-onset muscular dystrophies may be associated with profound loss of muscle function, affecting ambulation, posture, and cardiac and respiratory function. Lateonset muscular dystrophies may be mild and associated with slight weakness and inability to increase muscle mass., ${ }^{3,4}$

A better understanding of the molecular bases of MD has led to more accurate definitions of the clinical features and a new classification. Knowledge of disease-specific complications, implementation of anticipatory care, and medical advances have changed the standard of care, with an overall improvement in the clinical course, survival, and quality of life of affected people..$^{5-11}$

Muscular dystrophies can present an autosomal dominant, autosomal recessive, or Xlinked pattern of inheritance and can result from mutations affecting structural proteins localizable to the sarcolemma, nuclear or basement membrane, sarcomere, or non-structural enzymatic proteins..$^{1,4}$

In recent years, cardiac involvement has been observed in a growing number of genetic muscle diseases, and considerable progress has been made in understanding the relationships between disease skeletal muscle and cardiac muscle disease. ${ }^{12}$ Cardiologists may be unfamiliar with these diseases due to the low incidence; also, significant advances in respiratory care have only recently unmasked cardiomyopathy as a significant cause of death in MD. ${ }^{13,14}$ In several forms of MD, cardiac disease may even be the predominant manifestation of the underlying genetic myopathy.

Early detection of MD-associated cardiomyopathy is of considerable importance, as a prompt institution of cardio-protective medical therapies may slow adverse cardiac remodeling and attenuate heart failure symptoms in these patients. ${ }^{5-10}$

Electrocardiography (ECG) and echocardiography are typically advocated for screening, ${ }^{1,12}$ although very recently, cardiovascular magnetic resonance has shown promise in revealing early cardiac involvement when standard cardiac evaluation is unremarkable. ${ }^{15-17}$

This review will focus on the skeletal muscle diseases most commonly associated with cardiac complications that can be diagnosed by echocardiography, such as dystrophin-associated diseases or dystrophinopathies that include Duchenne (DMD) and Becker (BMD) muscular dystrophies, cardiomyopathy of DMD/BMD carriers and X-L dilated cardiomyopathy.

\section{Dystrophinopathic cardiomyopathy}

Interestingly, the first description of cardiac involvement associated with muscular dystrophy dates from 1836, by Gaetano Conte, a Neapolitan physician of the Santa Maria del Popolo degli Incurabili Hospital who, 32 years before the description of the disease by Duchenne de Boulogne, published in the journal Annali degli Incurabili, the case of a pseudohypertrophic myopathy in 2 brothers, one of them died with signs of heart hypertrophy..$^{18} \mathrm{~A}$ great contribution to the knowledge of myocardial damage in Duchenne and Becker muscular dystrophies is due to our group, who described the different clinical features of dystrophic cardiomyopathy in 1976, long before
Correspondence: Luisa Politano, Cardiomiologia e Genetica Medica - I Policlinico, Piazza Miraglia, 80138 Napoli, Italy.

E-mail: luisa.politano@unina2.it

Gerardo Nigro, Dipartimento di Cardiologia, Seconda, Università di Napoli (SUN), Italy.

E-mail: gerardo.nigro@unina2.it

Key words: cardiomyopathy, Duchenne muscular dystrophy, Becker muscular dystrophy, Duchenne and Becker carriers, genetics, echocardiography.

Received for publication: 30 July 2013

Revision received: 11 December 2013

Accepted for publication: 13 December 2013.

This work is licensed under a Creative Commons Attribution NonCommercial 3.0 License (CC BYNC 3.0).

(C) Copyright A. Palladino et al., 2013

Licensee PAGEPress, Italy

Cardiogenetics 2013; 3:e9

doi:10.4081/cardiogenetics.2013.e9

the discovery of the dystrophin gene and its localization in the heart. ${ }^{19}$ During the next two decades we documented the evolution of the myocardial damage, emphasizing the importance of the preclinical stage of the cardiomyopathy. ${ }^{20,21}$

The opportunity to follow several hundreds of patients affected by muscular dystrophies for more than three-four decades, allowed us to observe that cardiomyopathies, and especially those associated with muscular dystrophies, are constantly progressive, but evolve in a stage-wise manner, passing through a number of patho-physiologically distinct stages (Figure 1). Dilated cardiomyopathy and congestive heart failure are characteristic of dystrophinopathies but may represent the final stage of any type of cardiac involvement in muscular dystrophies.

Dystrophinopathic cardiomyopathy is caused by the complete/partial absence of dystrophin at the myocardial level, as a consequence of mutations in the dystrophin gene. Dystrophin gene is one of the more giant human genes so far identified. It comprises 8 promotors, 80 exons, a primary transcript of $14 \mathrm{~Kb}^{22}$ and a protein product of $427 \mathrm{Kd}$, composed by 3685 a. $\mathrm{a}^{23}$ (Figure 2). Mutations in the dystrophin gene can result in 4 different clinical pictures of dystrophinopathic cardiomyopathy: i) Duchenne cardiomyopathy; ii) Becker cardiomyopathy; iii) Carrier's cardiomyopathy; iv) X-linked dilated cardiomyopathy.

\section{Duchenne cardiomyopathy}

Cardiac involvement in DMD has long been recognized with initial pathology descriptions of myocyte hypertrophy and myocardial fibrosis, typical electrocardiographic abnormali- 
The severity of cardiac involvement increases with age towards stages of dilated cardiomyopathy and congestive heart failure..$^{28,30,31}$ Life expectancy is severely conditioned by the presence of the cardiomyopathy, closely related to the type of dystrophin gene mutation. ${ }^{32,33}$ Heart transplantation is often required. ${ }^{34}$

\section{Cardiomyopathy of Duchenne and}

Becker muscular dystrophies carriers

Cardiac involvement in DMD and BMD carriers was first described by our group in 1996 . $^{35}$ Cardiac abnormalities usually progress towards pictures of dilated cardiomyopathy, passing through stages of myocardial hypertrophy or dysrhythmias, as in DMD/BMD patients. Clinical features are often more severe than those observed in skeletal muscles, with an evolution similar to that of affected males, but delayed in time. We have recently shown that the onset of DMD phenotype in DMD carriers prevalently depends on the degree of the Xinactivation pattern. ${ }^{36}$ Often cardiomyopathy can be the main or the only manifestation of the carrier status. ${ }^{35,37}$ A percentage of carriers requires heart transplantation. ${ }^{38}$ Dystrophin anomalies can be detected at the membrane level of the myocytes on endomyocardial biopsy specimens (Figure 3 ). ${ }^{35,39}$

\section{X-linked dilated cardiomyopathy}

In this type of cardiomyopathy, described by Towbin in $1993,{ }^{40}$ the dystrophin defect is limited to the myocardium (Figure 3 ). The clinical picture is characterized by the onset of conges- tive heart failure in young boys, with a rapid fatal evolution within 2 years from the onset. Absence of muscle weakness is the rule, often associated with increased CK values. Cardiomyopathy in carriers is slowly progressive. Mutations responsible for X-linked cardiomyopathy are confined into two regions of the dystrophin gene: i) mutations at the 5 ' end of the gene, ${ }^{41}$ and ii) mutations in the spectrin-like dystrophin rod domain..$^{42}$ Mutations in NH2 domain seem to be associated to a more severe phenotype, ${ }^{32}$ because patients with these mutations are unable to compensate the lack of $\mathrm{M}$ isoform in the heart, upregulating the $\mathrm{B}$ and $\mathrm{P}$ isoforms of dystrophin, as happens in the muscle.

\section{Diagnosis of dystrophinopathic cardiomyopathy}

The diagnosis of dystrophinopathic cardiomyopathy relies on clinical and instrumental investigation. The preclinical stage can be diagnosed by minor ECG and echo signs. ECG signs include a classic tall right precordial $R$ wave, a $S / R$ ratio $>1$, a $P Q$ segment abnormally brief for the patient's age, a prolonged $Q T$ interval (after correction according to the heart rate), and a cardiomyopathic index $>4.6$ (Figure 4)..$^{21,28}$ Echocardiography can reveal areas of regional dyskinesia and fibrosis that can be detected by integrated back scattering (IBS) or echo-acoustic densitometry.

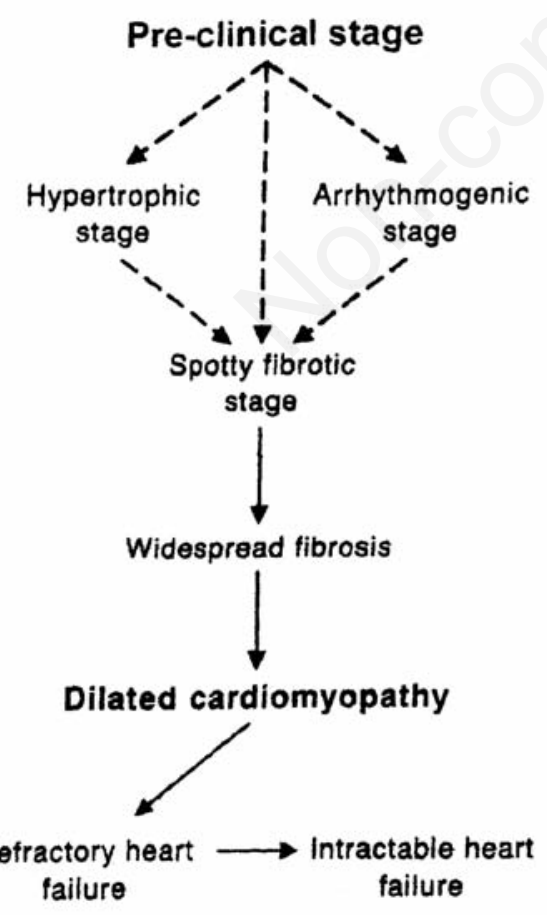

Figure 1. Schematic representation of dystrophinopathic cardiomyopathy and its stages.

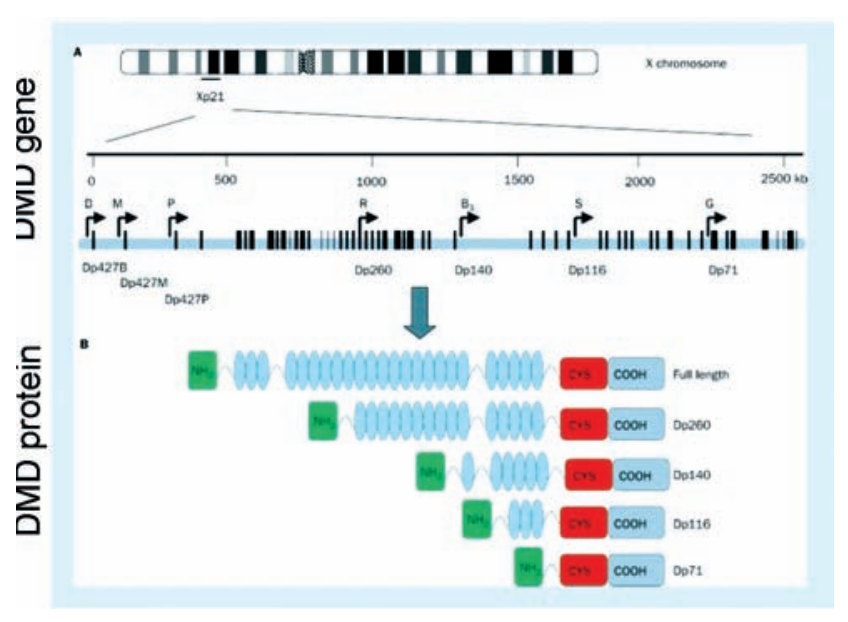

Figure 2. Schematic representation of dystrophyn gene and protein. 
During the intermediate stage that can manifest with the picture of hypertrophic or arrhythmogenic stage (Figure 1), echocardiography with IBS may show initial areas of fibrosis; echocardiography can also predict evolution toward a dilated stage by M-Modeechocardiography recorded at the level of the mitral valve, that shows an increased distance between the $\mathrm{E}$ point of the mitral valve and the left side of the ventricular septum. The hypertrophic aspects of this stage are easily detected by echocardiography. The width of the ventricular septum and the cardiac index (ratio thickness of ventricular septum/thickness of ventricular left free wall) are both increased (Figure 5). Echo and/or radionuclide investigation may also show areas of dyskinesia or akinesia caused by fibrosis. In these patients, frequent Holter monitoring will detect the onset of hyperkinetic arrhythmias that often appear during the night (Figure 6).

In the stage of dilated cardiomyopathy, clinical examination shows enlarged ventricles, reduced apical impulse, signs of decreased cardiac output, systolic murmur due to dilation of mitral annulus, distention of jugular veins, and hepatomegaly. Echo reveals dilated cardiac chambers, reduced ejection fraction $(<45 \%)$, reduced shortening fraction, and diffuse dyskinesia of the ventricular walls (Figure 7). The echo-Doppler cardiogram may indicate mild or severe mitral regurgitation (Figure 7). IBS shows diffuse areas of fibrosis in both ventricles. The ECG may show signs of fibrosis and various degrees of conduction defects (Figure 7). The transition from a spotty fibrosis to a widespread fibrosis may mimic a picture of myocardial infarction, with elevation of serum levels of troponin T, CK, CK-MB isoenzyme and lactate dehydrogenase, in young DMD boys. This picture can persist for a week and then disappears completely. ${ }^{43}$

The picture of a severe dilated cardiomyopathy with intractable cardiac failure is typical of Becker cardiomyopathy. BMD patients seem to pay a more benign muscle phenotype with a more severe cardiac evolution.

\section{Role of echocardiography in every day dystrophinopathic cardiomyopathy management}

ECG and echocardiography play a major role in the diagnostic and therapeutic setting of patients with dystrophinopathies. Electrocar diographic findings like short $\mathrm{PQ}$ and prolonged QT intervals on ECG, such as a picture of dilated cardiomyopathy of unknown origin in young women, should suggest a diagnosis of dystrophinopathic cardiomyopathy, especially when combined with the typical clinical presentation

\section{Duchenne \\ Cardiomyopathy}
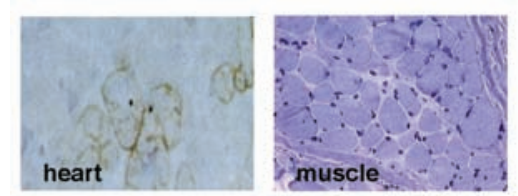

Becker Cardiomyopathy
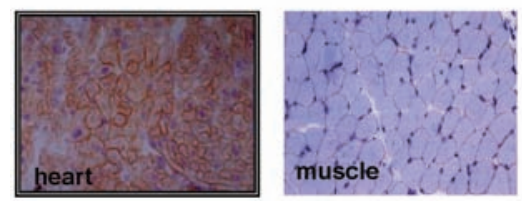

DMD/BMD carriers

Cardiomyopathy

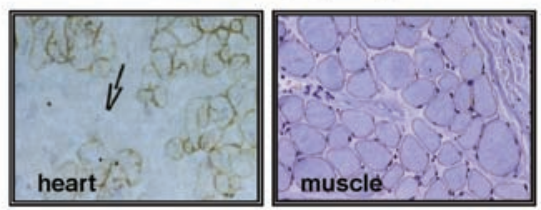

X-linked Dilated

Cardiomyopathy

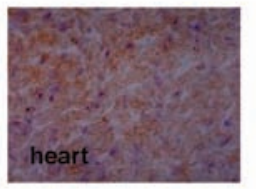

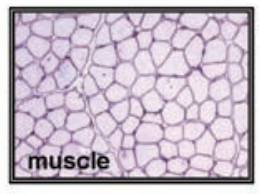

Figure 3. Immunostaining of dystrophin at muscle and cardiac level in the different types of dystrophinopathy. Top-left) Complete absence of dystrophin in heart and skeletal muscle in Duchenne muscular dystrophies (DMD); Bottom-left) Partial presence of dystrophin in heart and skeletal muscle in Becker muscular dystrophies (BMD). Top-right) Mosaic pattern of immunostaining of dystrophin in both heart and skeletal muscles in DMD/BMD carriers; Bottom-right) Complete absence of dystrophin immunostaining by anti-NH2 antibody in the heart (not in skeletal muscles) in X-linked dilated cardiomyopathy.

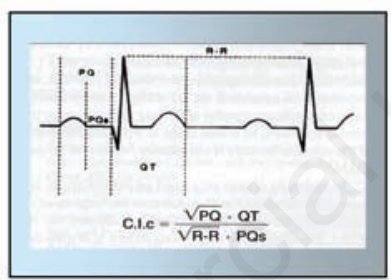

\section{ECG signs}

- Short $P Q$ segment

- Prolonged QT interval

- Cardiomyopathic Index $>4.6$

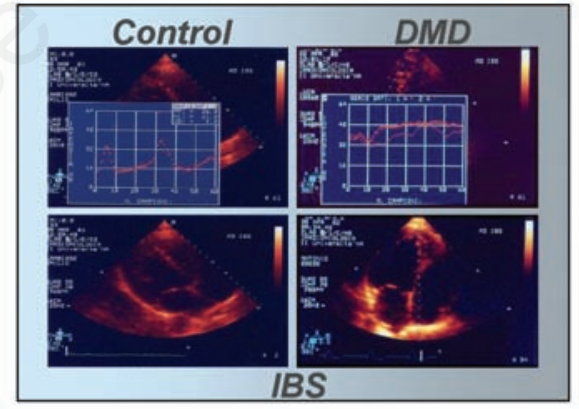

Echocardiographic signs
- Regional dyskinesia

- Regional fibrosis detected by

IBS (echo-acoustic densitometry)
Figure 4. Preclinical stage of dystrophinopathic cardiomyopathy: elettrocardiographic and echocardiographic signs.

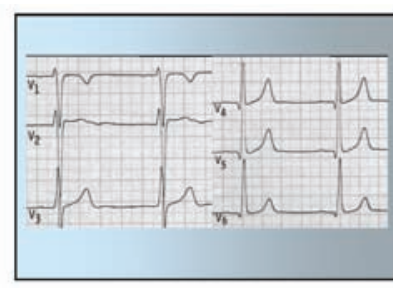

\section{ECG}

\section{- Signs of LV Hypertrophy}

- Deep S waves in right precordial leads

- Tall R waves in left precordial leads

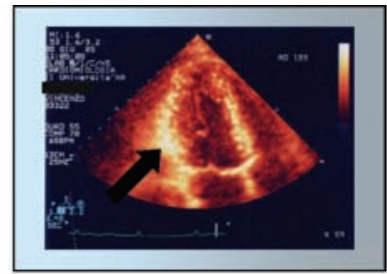

\section{Echocardiogram}

- Localized septal hypertrophy

- Ventricular hyper-contractility

- Out-flow gradient
Figure 5. Hypertrophic stage of dystrophinopathic cardiomyopathy: elettrocardiographic and echocardiographic signs. 
(walk anomalies and increased serum CK values). Echocardiography remains the most simple and accessible method to confirm the diagnosis and to assess the severity of the cardiomyopathy. Echocardiography is also useful to monitor the progression of the cardiomyopathy and the effects of a cardiological therapy.
Recent studies have suggested the presence of subclinical dysfunction in DMD patients using cardiac magnetic resonance imaging, tissue Doppler measurements, and myocardial velocity gradients. In particular, in young patients with DMD who have global normal systolic function, a reduction in systolic defor- mation parameters as well as reduced early diastolic myocardial velocities have been observed in the anterolateral and inferolateral LV walls. However, the prognostic significance of these findings warrants further longitudinal follow-up. ${ }^{4}$

\section{Prognosis of dystrophinopathic cardiomyopathy}

In Duchenne cardiomyopathy, sudden death caused by heart block or ventricular arrhythmias can occur starting from the intermediate stage of cardiomyopathy. However most Duchenne patients die of cardiorespiratory failure during the advanced or final stages of the disease. The prognosis is linked to respiratory treatment that includes long-term assisted ventilation, nocturnal ventilation and tracheostomy. It has been shown that ventilation by tracheostomy provides the better ventilatory support and, combined with the cardiological treatment, is able to prolong the life expectancy of these patients till the third-fourth decade. $^{11}$

The mean life expectancy of Becker patients is about $20 \%$ shorter compared with normal subjects. However the presence of cardiomyopathy greatly reduces life expectancy. After the onset of dilated cardiomyopathy, the prognosis worsens despite an appropriate cardiological treatment, so that only $50 \%$ of patients survive more than 5 years. Luckily, in the recent years a higher number of patients may have access to the waiting lists for heart transplantation. The natural history of X-L dilated cardiomyopathy is still unclear because the first stage of the disease is often asymptomatic. Similarly, the clinical course is unpredictable because the rate of progression to overt heart failure is not well known. However, by the time the patient becomes symptomatic, the prognosis is poor, with a 5-year mortality rate about 50\%. X-L dilated cardiomyopathy, like all primary dilated cardiomyopathies, is still the main indication for heart transplantation in both children and adults. The prognosis of dystrophinopathic cardiomyopathy in DMD/BMD carriers is influenced by the amount and degree of physical work, and by proportion of normal versus abnormal fibers in myocardium. $^{35,36}$

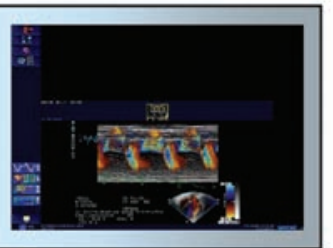

Figure 7. Dilated stage of dystrophinopathic cardiomyopathy: elettrocardiographic (ECG) and echocardiographic signs. A) ECG signs; B) Dilation of heart chambers: ejection fraction $<45 \%$, thin ventricular walls; C) and D) Mitral and tricuspid insufficiency.

C

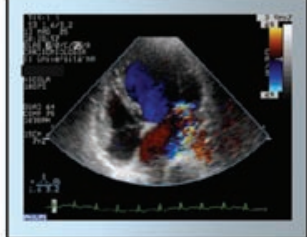

$$
\text { myocardium. } .^{35,36}
$$

\section{Treatment of dystrophinopathic cardiomyopathy}

In the early stages of dystrophinopathic cardiomyopathy, treatment with Ace-inhibitors is indicated, while anti-arrhythmic drugs are 
used to the onset of arrhythmias. It has been reported that the combination of Aceinhibitors and beta-blockers in patients with dystrophinopathies can delay the progression of left ventricular dysfunction and improve systolic function. In the advanced stages, standard therapy for heart failure with diuretics, digitalis and anticoagulants is adopted, without forgetting to correct electrolyte imbalances. ${ }^{10,45,46}$

\section{Conclusions}

Dystrophinopathic cardiomyopathy is an integral part of the clinical picture of Duchenne and Becker muscular dystrophies and can be the only marker of the dystrophin gene carrier status. About $90-95 \%$ of Duchenne patients present overt signs of dilated cardiomyopathy at the age of 18 years, while dilated cardiomyopathy represents an important factor limiting life expectancy in Becker patients and in X-linked dilated cardiomyopathy. Therefore we recommend that all DMD, BMD patients and DMD/BMD carriers, once molecularly diagnosed, are carefully monitored for the development of cardiomyopathy, even in the absence of overt cardiac or muscle signs/symptoms and in the early stages of the disease, using non-invasive cardiac techniques. In addition we hope for a closer cooperation between cardiologists and experts in cardio-myopathies to create a new figure, the cardio-myologists, with specific expertise and knowledge in all the diagnostic aspects and management of dystrophin heart muscle disorders.

\section{References}

1. Emery AE. The muscular dystrophies. Lancet 2002;359:687-95.

2. McNally EM, Pytel P. Muscle diseases: the muscular dystrophies. Annu Rev Pathol 2007;2:87-109.

3. Sewry CA. Muscular dystrophies: an update on pathology and diagnosis. Acta Neuropathol 2010;120:343-58.

4. Amato AA, Griggs RC. Overview of the muscular dystrophies. Handb Clin Neurol 2011;101:1-9.

5. Duboc D, Meune C, Lerebours G, et al. Effect of perindopril on the onset and progression of left ventricular dysfunction in Duchenne muscular dystrophy. J Am Coll Cardiol 2005;45:855-7.

6. Ishikawa Y, Bach JR, Minami R. Cardioprotection for Duchenne's muscular dystrophy. Am Heart J 1999;137:895-902.

7. Jefferies JL, Eidem BW, Belmont JW, et al. Genetic predictors and remodeling of dilated cardiomyopathy in muscular dystrophy.
Circulation 2005;112:2799-804.

8. Kajimoto H, Ishigaki K, Okumura K, et al. Beta-blocker therapy for cardiac dysfunction in patients with muscular dystrophy. Circ J 2006;70:991-4.

9. Ramaciotti C, Heistein LC, Coursey M, et al. Left ventricular function and response to enalapril in patients with duchenne muscular dystrophy during the second decade of life. Am J Cardiol 2006;98:825-7.

10. Politano L, Nigro G. Treatment of dystrophinopathic cardiomyopathy: review of the literature and personal results. Acta Myol 2012;31:24-30.

11. Passamano L, Taglia A, Palladino A, et al. Improvement of survival in Duchenne muscular dystrophy: retrospective analysis of 835 patients. Acta Myol 2012;31:121-5.

12. Bouhouch R, Elhouari T, Oukerraj L, et al. Management of cardiac involvement in neuromuscular diseases: review. Open Cardiovasc Med J 2008;2:93-6.

13. Bach JR. Update and perspective on noninvasive respiratory muscle aids. Part 2 : the expiratory aids. Chest 1994;105:1538-44.

14. Rideau Y, Politano L. Research against incurability. Treatment of lethal neuromuscular diseases focused on Duchenne muscular dystrophy. Acta Myol 2004;23:163-78.

15. Silva MC, Meira ZM, Gurgel Giannetti J, et al. Myocardial delayed enhancement by magnetic resonance imaging in patients with muscular dystrophy. J Am Coll Cardiol 2007;49:1874-79.

16. Yilmaz A, Gdynia HJ, Baccouche $\mathrm{H}$, et al. Cardiac involvement in patients with Becker muscular dystrophy: new diagnostic and pathophysiological insights by a CMR approach. J Cardiovasc Magn Reson 2008;10:50.

17. Verhaert D, Richards K, Rafael-Fortney JA, Raman SV. Cardiac involvement in patients with muscular dystrophies: magnetic resonance imaging phenotype and genotypic considerations. Circ Cardiovasc Imaging 2011;4:67-76.

18. Conte G, Gioia L. Scrofola del sistema muscolare. Ann Clin Osped Incurab Napoli 1836;2:66-79.

19. Nigro G, Limongelli FM, Comi LI, et al. The cardiomyopathy in progressive muscular dystrophy. Patologia Umana 1976;1:259-67.

20. Nigro G, Comi LI, Politano et al. Electrocardiographic evaluation of P-type stage of dystrophic cardiomyopathy. Cardiomyology 1984;3:45-58.

21. Nigro G, Comi LI, Politano L, Bain RJI. The incidence and evolution of cardiomyopathy in Duchenne muscular dystrophy. Intern J Cardiol 1990;26:271-7.

22. Monaco AP, Neve RL, Colletti-Feener C, et al. Isolation of candidate cDNAs for portions of the Duchenne muscular dystrophy gene. Nature 1986;323:646-50.

23. Hoffman EP, Brown RH Jr, Kunkel LM. Dystrophin: the protein product of the Duchenne muscular dystrophy locus. Cell 1987;51:919-28.

24. Manning GW, Cropp GJ. The electrocardiogram in progressive muscular dystrophy. Br Heart J 1958;3:416.

25. Perloff JK, Roberts WC, de Leon AC, et al. Distinctive electrocardiography of Duchenne's progressive muscular dystrophy: an elettrocardiographic-pathologic correlative study. Am J Med 1967;42:179-8.

26. Kovick RB, Fogelman AM, Abbasi AD, et al. Echocardiographic evaluation of posterior left ventricular wall motion in muscular dystrophy. Circulation 1975;52:447-54.

27. Ahmad M, Sanderson JE, Dubowitz V, et al. Echocardiographic assessment of left ventricular function in Duchenne's muscular dystrophy. Br Heart J 1978;40:734-40.

28. Nigro G, Comi LI, Politano L, Nigro GE. Cardiomyopathies associated with muscular dystrophies. In: Engel AG and FranziniArmstrong C, eds. Myology. New York: McGraw-Hill; 2004. pp 1239-1256.

29. Bello L, Piva L, Barp A, et al. Importance of SPP1 genotype as a covariate in clinical trials in Duchenne muscular dystrophy. Neurology 2012;79:159-62.

30. Nigro G, Comi LI, Politano L, et al: Evaluation of the cardiomyopathy in Becker muscular dystrophy. Muscle Nerve 1995;18:283-91.

31. Hoogerwaard EM, de Voogt WG, Wilde AA, et al. Evolution of cardiac abnormalities in Becker muscular dystrophy over a 13-year period. J Neurol 1997;244:657-63.

32. Arbustini E, Diegoli M, Morbini P, et al. Prevalence and characteristics of the dystrophin defects in adult male patients with dilated cardiomyopathy. J Am Coll Cardiol 2000;35:1760-8.

33. Nigro G, Politano L, Nigro V, et al. Mutations in dystrophin gene and cardiomyopathy. Neuromusc Disord 1994;4: 371-9.

34. Piccolo G, Azan G, Tonin P, et al. Dilated cardiomyopathy requiring cardiac transplantation as initial manifestation of Xp21 Becker type muscular dystrophy. Neuromusc Disord 1994;4:143-6.

35. Politano L, Nigro V, Nigro G, et al. Development of cardiomyopathy in female carriers of Duchenne and Becker muscular dystrophies. JAMA 1996;275:1335-8.

36. Viggiano E, Picillo E, Cirillo A, Politano L. Comparison of X-chromosome inactivation in Duchenne muscle/myocardium-manifesting carriers, non-manifesting carriers and related daughters. Clin Genet 2013;84:265-70.

37. Hoogerwaard EM, van der Wouw PA, Wilde AAM, et al. Cardiac involvement in carriers 
of Duchenne and Becker muscular dystrophy. Neuromusc Disord 1999;9:347-51.

38. Melacini P, Fanin M, Angelini A, et al. Cardiac transplantation in a Duchenne muscular dystrophy carrier. Neuromuscul Disord 1998;8:585-90.

39. Nigro G, Di Somma S, Comi LI, et al. Structural basis of cardiomyopathy in Duchenne/Becker carriers. Endomyocardial biopsy evaluation. Ann N Y Acad Sci 1995;752:108-10.

40. Towbin JA, Hejtmancik JF, Brink P, et al. Xlinked dilated cardiomyopathy. Molecular genetic evidence of linkage to the Duchenne muscular dystrophy (dys- trophin) gene at the Xp21 locus. Circulation 1993;87:1854-65.

41. Muntoni F, Cau M, Ganau A, et al. Brief report: deletion of the dystrophin musclepromoter region associated with X-linked dilated cardiomyopathy. N Engl J Med 1993;329:921-5.

42. Ferlini A, Sewry C, Melis MA, et al. Xlinked dilated cardiomyopathy and the dystrophin gene. Neuromuscul Disord 1999;9:339-46.

43. Politano L, Palladino A, Petretta VR, et al. ST-segment displacement in Duchenne muscular dystrophy: myocardial necrosis or apoptosis? Acta Myol 2003;22:5-10.
44. Mertens L, Ganame J, Claus P, et al. Early regional myocardial dysfunction in young patients with Duchenne muscular dystrophy. Am Soc Echocardiogr 2008;21:104954.

45. Ogata H, Ishikawa Y, Ishikawa Y, Minami R. Beneficial effects of beta-blockers and angiotensin-converting enzyme inhibitors in Duchenne muscular dystrophy. J Cardiol 2009;53:72-8.

46. Viollet L, Thrush PT, Flanigan KM, et al. Effects of angiotensin-converting enzyme inhibitors and/or beta blockers on the cardiomyopathy in Duchenne muscular dystrophy. Am J Cardiol 2012;110:98-102. 